\title{
HELICOIDAL SURFACES IN THE THREE DIMENSIONAL SIMPLY ISOTROPIC SPACE $\square_{3}^{1}$
}

\author{
MURAT KEMAL KARACAN, DAE WON YOON AND SEZAI KIZILTUG
}

\begin{abstract}
In this paper, we classify helicoidal surfaces in the three dimensional simply isotropic space $\mathbb{1}_{3}^{1}$ satisfying some algebraic equations in terms of the coordinate functions and the Laplacian operators with respect to the first, the second and the third fundamental form of the surface. We also give explicit forms of these surfaces.
\end{abstract}

\section{Introduction}

Let $\mathbf{x}: \mathbf{M} \rightarrow \mathbb{E}^{m}$ be an isometric immersion of a connected $n$-dimensional manifold in the $m$-dimensional Euclidean space $\mathbb{E}^{m}$. Denote by $\mathbf{H}$ and $\Delta$ the mean curvature and the Laplacian of $\mathbf{M}$ with respect to the Riemannian metric on $\mathbf{M}$ induced from that of $\mathbb{E}^{m}$, respectively. Takahashi ([17]) proved that the submanifolds in $\mathbb{E}^{m}$ satisfying $\Delta \mathbf{x}=\lambda \mathbf{x}$, that is, all coordinate functions are eigenfunctions of the Laplacian with the same eigenvalue $\lambda \in \mathbb{R}$ are either the minimal submanifolds of $\mathbb{E}^{m}$ or the minimal submanifolds of hypersphere $\mathbb{S}^{m-1}$ in $\mathbb{E}^{m}$.

As an extension of Takahashi theorem, Garay studied in [11] hypersurfaces in $\mathbb{E}^{m}$ whose coordinate functions are eigenfunctions of the Laplacian, but not necessarily associated to the same eigenvalue. He considered hypersurfaces in $\mathbb{E}^{m}$ satisfying the condition

$$
\Delta \mathbf{x}=\mathbf{A x}
$$

where $\mathbf{A} \in \operatorname{Mat}(m, \mathbb{R})$ is an $m \times m$-diagonal matrix, and proved that such hypersurfaces are minimal $(\mathbf{H}=0)$ in $\mathbb{E}^{m}$ and open pieces of either round hyperspheres or generalized right spherical cylinders.

Related to this, Dillen, Pas and Verstraelen ([9]) investigated surfaces in $\mathbb{E}^{3}$ whose immersions satisfy the condition

$$
\Delta \mathbf{x}=\mathbf{A x}+\mathbf{B},
$$

Received April 27, 2016, accepted October 19, 2016.

2010 Mathematics Subject Classification. 53A35, 53A40, 53B30.

Key words and phrases. Helicoidal surfaces, Laplacian operator, simply isotropic space.

Corresponding author: Murat Kemal Karacan. 
where $\mathbf{A} \in \operatorname{Mat}(3, \mathbb{R})$ is a $3 \times 3$-real matrix and $\mathbf{B} \in \mathbb{R}^{3}$. In other words, each coordinate function is of 1-type in the sense of Chen ([8]). For the Lorentzian version of surfaces satisfying (1.2), Alias, Ferrandez and Lucas ([1]) proved that the only such surfaces are minimal surfaces and open pieces of Lorentz circular cylinders, hyperbolic cylinders, Lorentz hyperbolic cylinders, hyperbolic spaces or pseudo-spheres.

The notion of an isometric immersion $\mathbf{x}$ is naturally extended to smooth functions on submanifolds of Euclidean space or pseudo-Euclidean space. The most natural one of them is the Gauss map of the submanifold. In particular, if the submanifold is a hypersurface, the Gauss map can be identified with the unit normal vector field to it. Dillen, Pas and Verstraelen ([10]) studied surfaces of revolution in the three dimensional Euclidean space $\mathbb{E}^{3}$ such that its Gauss map G satisfies the condition

$$
\Delta \mathbf{G}=\mathbf{A G}
$$

where $\mathbf{A} \in \operatorname{Mat}(3, \mathbb{R})$. Baikoussis and Verstraelen ([4]) studied the helicoidal surfaces in $\mathbb{E}^{3}$. Choi ([6]) completely classified the surfaces of revolution satisfying the condition (1.3) in the three dimensional Minkowski space $\mathbb{E}_{1}^{3}$. The authors $([7,18])$ classified surfaces of revolution satisfying (1.2) and (1.3) in the three dimensional Minkowski space and pseudo-Galilean space. Baba-Hamen and Bekkar ([12]) classified the helicoidal surfaces in the 3-dimensional Lorentz-Minkowski space under the condition

$$
\Delta \mathbf{x}^{i}=\lambda^{i} x^{i}
$$

where $\lambda^{i} \in \mathbb{R}$.The authors ([13]) classified surfaces of revolution satisfying

$$
\Delta^{\mathrm{III}} \mathbf{r}=\mathbf{A r},
$$

in the three dimensional Lorentz-Minkowski space.

The main purpose of this paper is to complete classification of helicoidal surfaces in the three dimensional simply isotropic space $\square_{3}^{1}$ in terms of the position vector field and the Laplacian operator.

\section{Preliminaries}

A simply isotropic space $\mathbb{1}_{3}^{1}$ is a Cayley-Klein space defined from the three dimensional projective space $\mathscr{P}\left(\mathbb{R}^{3}\right)$ with the absolute figure which is an ordered triple $\left(w, f_{1}, f_{2}\right)$, where $w$ is a plane in $\mathscr{P}\left(\mathbb{R}^{3}\right)$ and $f_{1}, f_{2}$ are two complex-conjugate straight lines in $w$. The homogeneous coordinates in $\mathscr{P}\left(\mathbb{R}^{3}\right)$ are introduced in such a way that the absolute plane $w$ is given by $x_{0}=0$ and the absolute lines $f_{1}, f_{2}$ by $x_{0}=x_{1}+i x_{2}=0, x_{0}=x_{1}-i x_{2}=0$. The intersection point $\mathbb{F}(0: 0: 0: 1)$ of these two lines is called the absolute point. The group of motions of the simply 
isotropic space is a six-parameter group given in the affine coordinates $x=\frac{x_{1}}{x_{0}}, y=\frac{x_{2}}{x_{0}}, z=\frac{x_{3}}{x_{0}}$ by

$$
\begin{aligned}
& \bar{x}=a+x \cos \theta-y \sin \theta \\
& \bar{y}=b+x \sin \theta+y \cos \theta \\
& \bar{z}=c+c_{1} x+c_{2} y+z,
\end{aligned}
$$

where $a, b, c, c_{1}, c_{2}, \theta \in \mathbb{R}$. Such affine transformations are called isotropic congruence transformations or $i$-motions [12].

Isotropic geometry has different types of lines and planes with respect to the absolute figure. A line is called non-isotropic (resp. completely isotropic) if its point at infinity does not coincide (coincides) with the point $\mathbb{F}$. A plane is called non-isotropic (resp. isotropic) if its line at infinity does not contain $\mathbb{F}$. Completely isotropic lines and isotropic planes in this affine model appear as vertical, i.e., parallel to the $z$-axis. Finally, the metric of the simply isotropic space $\square_{3}^{1}$ is given by

$$
d s^{2}=d x^{2}+d y^{2}
$$

A surface $\mathbf{M}$ immersed in $\rrbracket_{3}^{1}$ is called admissible if it has no isotropic tangent planes. For such a surface, the coefficients $E, F, G$ of its first fundamental form are calculated with respect to the induced metric and the coefficients $L, M, N$ of the second fundamental form, with respect to the normal vector field of a surface which is always completely isotropic. The (isotropic) Gaussian and mean curvature are defined by

$$
\mathbf{K}=k_{1} k_{2}=\frac{L N-M^{2}}{E G-F^{2}}, \quad 2 \mathbf{H}=k_{1}+k_{2}=\frac{E N-2 F M+G L}{E G-F^{2}},
$$

where $k_{1}, k_{2}$ are principal curvatures, i.e., extrema of the normal curvature determined by the normal section (in completely isotropic direction) of a surface. Since $E G-F^{2}>0$, for the function in the denominator we often put $W^{2}=E G-F^{2}$. The surface $\mathbf{M}$ is said to be isotropic flat (resp. isotropic minimal ) if $\mathbf{K}$ (resp.H) vanishes [3, 15, 16].

It is well known in terms of local coordinates $\{u, v\}$ of $\mathbf{M}$ the Laplacian operators $\Delta^{\mathbf{I}}, \Delta^{\mathbf{I I}}$, $\Delta^{\mathrm{III}}$ of the first, the second and the third fundamental form on $\mathbf{M}$ are defined by $([5,13,14])$

$$
\begin{gathered}
\Delta^{\mathbf{I}} \mathbf{x}=-\frac{1}{\sqrt{\left|E G-F^{2}\right|}}\left[\frac{\partial}{\partial u}\left(\frac{G \mathbf{x}_{u}-F \mathbf{x}_{v}}{\sqrt{\left|E G-F^{2}\right|}}\right)-\frac{\partial}{\partial v}\left(\frac{F \mathbf{x}_{u}-E \mathbf{x}_{v}}{\sqrt{\left|E G-F^{2}\right|}}\right)\right], \\
\Delta^{\mathbf{I I}} \mathbf{x}=-\frac{1}{\sqrt{\left|L N-M^{2}\right|}}\left[\frac{\partial}{\partial u}\left(\frac{N \mathbf{x}_{u}-M \mathbf{x}_{v}}{\sqrt{\left|L N-M^{2}\right|}}\right)-\frac{\partial}{\partial v}\left(\frac{M \mathbf{x}_{u}-L \mathbf{x}_{v}}{\sqrt{\left|L N-M^{2}\right|}}\right)\right],
\end{gathered}
$$

and 
where

$$
\Delta^{\mathrm{III}} \mathbf{x}=-\frac{\sqrt{E G-F^{2}}}{L N-M^{2}}\left[\begin{array}{c}
\frac{\partial}{\partial u}\left(\frac{Z \mathbf{x}_{u}-Y \mathbf{x}_{v}}{\left(L N-M^{2}\right) \sqrt{E G-F^{2}}}\right)- \\
\frac{\partial}{\partial v}\left(\frac{Y \mathbf{x}_{u}-X \mathbf{x}_{\nu}}{\left(L N-M^{2}\right) \sqrt{E G-F^{2}}}\right)
\end{array}\right],
$$

$$
\begin{aligned}
& X=E M^{2}-2 F L M+G L^{2}, \\
& Y=E M N-F L N+G L M-F M^{2}, \\
& Z=G M^{2}-2 F N M+E N^{2} .
\end{aligned}
$$

\section{Helicoidal surfaces in $\rrbracket_{3}^{1}$}

Now we adapt the above notion to isotropic spaces. Considering the $i$-motions given by (2.1), the Euclidean rotation in the simply isotropic space $\square_{3}^{1}$ given by the normal form (in affine coordinates)

$$
\begin{aligned}
& \bar{x}=x \cos \theta-y \sin \theta \\
& \bar{y}=x \sin \theta+y \cos \theta \\
& \bar{z}=z,
\end{aligned}
$$

where $\theta \in \mathbb{R}[2]$.

First of all, we consider a plane curve $\alpha$ parametrized by $\alpha(u)=(f(u), 0, g(u))$ or isotropic curve $\alpha(u)=(0, f(u), g(u))$ around the $z$-axis by Euclidean rotation (3.1), where $g$ is a positive function and $f$ is a smooth function on an open interval $I$. Then by $i$-motion, the helicoidal surface $\mathbf{M}$ can be written as

$$
\mathbf{x}(u, v)=(f(u) \cos v, g(u) \sin v, g(u)+h v)
$$

or

$$
\mathbf{x}(u, v)=(-f(u) \sin v, f(u) \cos v, g(u)+h v)
$$

for any $h \in \mathbb{R}$. We will use the parametrization of the unit speed profile curve $\alpha(u)=(u, 0, g(u))$ [2].

\section{Helicoidal Surfaces Satisfying $\Delta^{\mathrm{I}} \mathbf{x}_{i}=\lambda_{i} \mathbf{x}_{i}$}

In this section, we classify helicoidal surfaces given by (3.2) in $\mathbb{q}_{3}^{1}$ satisfying the equation

$$
\Delta^{\mathbf{I}} \mathbf{x}_{i}=\lambda_{i} \mathbf{x}_{i},
$$

where $\lambda_{i} \in \mathbb{R}, i=1,2,3$ and

$$
\Delta^{\mathbf{I}} \mathbf{x}=\left(\Delta^{\mathbf{I}} \mathbf{x}_{1}, \Delta^{\mathbf{I}} \mathbf{x}_{2}, \Delta^{\mathbf{I}} \mathbf{x}_{3}\right),
$$


where

$$
\mathbf{x}_{1}=u \cos v, \mathbf{x}_{2}=u \sin v, \mathbf{x}_{3}=g(u)+h v .
$$

For this helicoidal surface, the coefficients of the first and second fundamental form are, respectively

$$
\begin{aligned}
& E=1, \quad F=0, \quad G=u^{2}, \\
& L=g^{\prime \prime}, \quad M=-\frac{h}{u}, \quad N=u g^{\prime} .
\end{aligned}
$$

The Gaussian curvature $\mathbf{K}$ and the mean curvature $\mathbf{H}$ are

$$
\mathbf{K}=\frac{-h^{2}+u^{3} g^{\prime}(u) g^{\prime \prime}(u)}{u^{4}}, \mathbf{H}=\frac{g^{\prime}(u)+u g^{\prime \prime}(u)}{2 u},
$$

respectively.

Corollary 1. Helicoidal surfaces given by (3.2) in the three dimensional simply isotropic space $\square_{3}^{1}$ are isotropic flat $(\mathbf{K}=0)$, if

$$
g(u)= \pm\left\{h \arctan \left(\frac{h}{\sqrt{-h^{2}+2 c_{1} u^{2}}}\right)+\sqrt{-h^{2}+2 c_{1} u^{2}}+c_{2}\right\}
$$

for some constants $c_{1}$ and $c_{2}$.

Proof. From (4.4), if $\mathbf{K}=0$, then we have

$$
u^{3} g^{\prime}(u) g^{\prime \prime}(u)=h^{2}
$$

If we solve this equation, we obtain

$$
g(u)= \pm\left\{h \arctan \left(\frac{h}{\sqrt{-h^{2}+2 c_{1} u^{2}}}\right)+\sqrt{-h^{2}+2 c_{1} u^{2}}+c_{2}\right\} .
$$

Corollary 2. Helicoidal surfaces given by (3.2) in the three dimensional simply isotropic space $\square_{3}^{1}$ have isotropic constant mean curvature if $g(u)=c_{1} \ln u+a \frac{u^{2}}{2}+c_{2}$, where $a, c_{1}, c_{2}$ are constants. If $a=0$, then helicoidal surfaces are isotropic minimal.

Proof. From (4.4), if helicoidal surface given by (3.2) which is isotropic minimal, then $\mathbf{H}=0$. Thus we get $g^{\prime}(u)+u g^{\prime \prime}(u)=0$. So we obtain $g(u)=c_{1} \ln u+c_{2}$ for some constants $c_{1}, c_{2}$. If helicoidal surface given by (3.2) which is CMC surface, then $\mathbf{H}=a$, where $a \in \mathbb{R}$. From (4.4), we have

$$
\frac{g^{\prime}(u)+u g^{\prime \prime}(u)}{2 u}=a
$$

Thus we obtain

$$
g(u)=c_{1} \ln u+a \frac{u^{2}}{2}+c_{2} .
$$


Suppose that the surface has non zero Gaussian curvature, so $-h^{2}+u^{3} g^{\prime}(u) g^{\prime \prime}(u) \neq 0$, $\forall u \in I$. By a straightforward computation, the Laplacian operator on $\mathbf{M}$ with the help of (4.2) and (2.3) turns out to be

$$
\Delta^{\mathbf{I}} \mathbf{x}=\left(0,0,-\frac{g^{\prime}(u)+u g^{\prime \prime}(u)}{u}\right) .
$$

Suppose that M satisfies (4.1). Then, we have

$$
\begin{aligned}
0 & =\lambda_{1}(u \cos v), \\
0 & =\lambda_{2}(u \sin v), \\
-\frac{g^{\prime}(u)+u g^{\prime \prime}(u)}{u} & =\lambda_{3}(g(u)+h v) .
\end{aligned}
$$

where $\lambda_{1}, \lambda_{2}$ and $\lambda_{3} \in \mathbb{R}$. This means that $\mathbf{M}$ is at most of 1-type. Thus we have $\lambda_{3}=0$, which implies the mean curvature $\mathbf{H}$ vanishes identically because of (4.6). Therefore, the helicoidal surfaces are isotropic minimal.

Corollary 3. Helicoidal surface given by (3.2) in the three dimensional simply isotropic space $\square_{3}^{1}$ is harmonic if and only if the surface $\mathbf{M}$ is isotropic minimal.

The corollary is well-know fact because, in general,

$$
\Delta^{\mathrm{I}} \mathbf{x}=-2 \overrightarrow{\mathbb{M}}=-2 \mathbf{H} \mathbb{N}, \Delta^{\mathrm{I}} \mathbf{x}=0
$$

$(\mathbf{M}=$ harmonic $) \Leftrightarrow \mathbf{H}=0$. In this paper,

$$
\Delta^{\mathbf{I}} \mathbf{x}=-2\left(0,0, \frac{g^{\prime}(u)+u g^{\prime \prime}(u)}{2 u}\right)=-2\left(\frac{g^{\prime}(u)+u g^{\prime \prime}(u)}{2 u}\right)(0,0,1)=-2 \mathbf{H} \mathbb{N} .
$$

\section{Helicoidal surfaces satisfying $\Delta^{\mathrm{II}} \mathbf{x}_{i}=\lambda_{i} \mathbf{x}_{i}$}

In this section, we classify helicoidal surfaces with non-degenerate second fundamental form in $\square_{3}^{1}$ satisfying the equationIn

$$
\Delta^{\mathrm{II}} \mathbf{x}_{i}=\lambda_{i} \mathbf{x}_{i}
$$

where $\lambda_{i} \in \mathbb{R}, i=1,2,3$ and

$$
\Delta^{\mathrm{II}} \mathbf{x}=\left(\Delta^{\mathrm{II}} \mathbf{x}_{1}, \Delta^{\mathrm{II}} \mathbf{x}_{2}, \Delta^{\mathrm{II}} \mathbf{x}_{3}\right),
$$

where

$$
\mathbf{x}_{1}=u \cos v, \mathbf{x}_{2}=u \sin v, \mathbf{x}_{3}=g(u)+h v,
$$


where $\lambda_{i} \in \mathbb{R}, i=1,2,3$. In this case, the function $g$ is non constant, everywhere. By a straightforward computation, the Laplacian operator on $\mathbf{M}$ with the help of (4.2), (4.4) and (2.4) turns out to be

$$
\Delta^{\mathbf{I I}} \mathbf{x}=\left(\begin{array}{c}
A(u)\left(-h u \sin v+u^{2} g^{\prime}(u) \cos v\right) \\
A(u)\left(h u \cos v+u^{2} g^{\prime}(u) \sin v\right) \\
B(u)
\end{array}\right),
$$

where

$$
\begin{gathered}
A(u)=\frac{1}{2\left(h^{2}-u^{3} g^{\prime} g^{\prime \prime}\right)^{2}}\left(4 h^{2}-u^{3} g^{\prime} g^{\prime \prime}+u^{4} g^{\prime \prime^{2}}+u^{4} g^{\prime} g^{\prime \prime \prime}\right), \\
B(u)=\frac{u^{2} g^{\prime}}{2\left(h^{2}-u^{3} g^{\prime} g^{\prime \prime}\right)^{2}}\left(4 h^{2} g^{\prime}+4 h^{2} u g^{\prime \prime}-u^{3} g^{\prime^{2}} g^{\prime \prime}-3 u^{4} g^{\prime} g^{\prime \prime^{2}}+u^{4} g^{\prime^{2}} g^{\prime \prime \prime}\right) .
\end{gathered}
$$

We observe that

$$
B(u)=\frac{u^{2} g^{\prime^{2}}}{2\left(h^{2}-u^{3} g^{\prime} g^{\prime \prime}\right)^{2}} A(u)-\frac{4 u^{2} g^{\prime^{2}}}{2\left(h^{2}-u^{3} g^{\prime} g^{\prime \prime}\right)^{2}}\left(h^{2} u g^{\prime \prime}-u^{4} g^{\prime} g^{\prime \prime^{2}}\right) .
$$

Equation (5.1) by means of (5.2) rise to the following system of ordinary differential equations

$$
\begin{aligned}
\Delta^{\mathbf{I I}}(u \cos v) & =\lambda_{1}(u \cos v), \\
\Delta^{\mathbf{I I}}(u \sin v) & =\lambda_{2}(u \sin v), \\
\Delta^{\mathbf{I I}}(g(u)+h v) & =\lambda_{3}(g(u)+h v),
\end{aligned}
$$

where $\lambda_{1}, \lambda_{2}$ and $\lambda_{3} \in \mathbb{R}$. This means that $\mathbf{M}$ is at most of 3- type. Equation (5.6) becomes

$$
\begin{aligned}
A(u)\left(-h u \sin v+u^{2} g^{\prime}(u) \cos v\right) & =\lambda_{1}(u \cos v), \\
A(u)\left(h u \cos v+u^{2} g^{\prime}(u) \sin v\right) & =\lambda_{2}(u \sin v), \\
B(u) & =\lambda_{3}(g(u)+h v) .
\end{aligned}
$$

First of all, we assume that $\mathbf{M}$ satisfies the condition $\Delta^{\mathrm{II}} \mathbf{x}=0$, thus we have $\lambda_{1}=\lambda_{2}=\lambda_{3}=0$. In this case (5.7) can be written as

$$
\begin{aligned}
A(u)\left(-h u \sin v+u^{2} g^{\prime}(u) \cos v\right) & =0, \\
A(u)\left(h u \cos v+u^{2} g^{\prime}(u) \sin v\right) & =0, \\
B(u) & =0 .
\end{aligned}
$$

Since the functions $\cos v, \sin v$ and the constant function are linearly independent, by (5.5) and (5.8), we get

$$
A(u)=0,
$$




$$
B(u)=0
$$

or

$$
\begin{gathered}
\left(4 h^{2}-u^{3} g^{\prime} g^{\prime \prime}+u^{4} g^{\prime^{2}}+u^{4} g^{\prime} g^{\prime \prime \prime}\right)=0, \\
-4 u^{3} g^{\prime^{2}}=0 .
\end{gathered}
$$

There are no any suitable solutions of (5.9). Differential equation (5.10) admits the particular solution $g(u)=c_{1}$, where $c_{1} \in \mathbb{R}$. In this case, $\mathbf{M}$ is parametrized by

$$
\mathbf{x}(u, v)=\left(u \cos v, u \sin v, c_{1}+h v\right) .
$$

From (5.7), we easly get

$$
A(u)=\frac{\lambda_{1}(u \cos v)}{\left(-h u \sin v+u^{2} g^{\prime}(u) \cos v\right)}
$$

and

$$
A(u)=\frac{\lambda_{2}(u \sin v)}{\left(h u \cos v+u^{2} g^{\prime}(u) \sin v\right)} .
$$

We discuss two cases according to the values of $\lambda_{i}$.

Case 1: Let $\lambda_{1}=0$ or $\lambda_{2}=0, \lambda_{3} \neq 0$, from (5.5), we have

$$
-\frac{2 u}{\left(h^{2}-u^{3} g^{\prime} g^{\prime \prime}\right)}=\lambda_{3}(g(u)+h v) \text {. }
$$

There are no any suitable solutions of (5.13).

Case 2: If $\lambda_{1} \neq 0$ or $\lambda_{2} \neq 0, \lambda_{3}=0$, from (5.5), we have

$$
\frac{\lambda_{1}(\cos v)}{\left(-h \sin v+u g^{\prime}(u) \cos v\right)}-4\left(h^{2} g^{\prime \prime}-u^{3} g^{\prime} g^{\prime \prime^{2}}\right)=0
$$

and

$$
\frac{\lambda_{2}(\sin v)}{\left(h \cos v+u g^{\prime}(u) \sin v\right)}-4\left(h^{2} g^{\prime \prime}-u^{3} g^{\prime} g^{\prime \prime^{2}}\right)=0 .
$$

There are no any suitable solutions of (5.14) and (5.15).

Definition 1. A surface of in the three dimensional simple isotropic space is said to be IIharmonic if it satisfies the condition $\Delta^{\mathrm{II}} \mathbf{x}=\mathbf{0}$.

Corollary 4. Helicoidal surface given by (3.2) in the three dimensional simply isotropic space $\square_{3}^{1}$ is II-harmonic surface if $g(u)=c_{1}$ for some $c_{1} \in \mathbb{R}$.

Theorem 1 (Classification). Let $\mathbf{M}$ be a non $\mathbf{I I}$-harmonic helicoidal surfaces with non-degenerate second fundamental form given by (3.2) in the three dimensional simply isotropic space $\square_{3}^{1}$. There are no the surfaces $\mathbf{M}$ satisfies the condition $\Delta^{\mathbf{I}} \mathbf{x}_{i}=\lambda_{i} \mathbf{x}_{i}$, where $\lambda_{i} \in \mathbb{R}, i=1,2,3$. 
6. Helicoidal surfaces satisfying $\Delta^{\mathrm{III}} \mathbf{x}_{i}=\lambda_{i} \mathbf{x}_{i}$

In this section, we classify helicoidal surfaces with non-degenerate second fundamental form in $\mathbb{\square}_{3}^{1}$ satisfying the equation

$$
\Delta^{\mathrm{III}} \mathbf{x}_{i}=\lambda_{i} \mathbf{x}_{i}
$$

where $\lambda_{i} \in \mathbb{R}, i=1,2,3$ and

$$
\Delta^{\mathrm{III}} \mathbf{x}=\left(\Delta^{\mathrm{III}} \mathbf{x}_{1}, \Delta^{\mathrm{III}} \mathbf{x}_{2}, \Delta^{\mathrm{III}} \mathbf{x}_{3}\right),
$$

where

$$
\mathbf{x}_{1}=u \cos v, \mathbf{x}_{2}=u \sin v, \mathbf{x}_{3}=g(u)+h v,
$$

By a straightforward computation, the Laplacian operator on $\mathbf{M}$ with the help of (4.3) and (2.5) turns out to be

$$
\Delta^{\mathrm{III}} \mathbf{x}=\left(\begin{array}{c}
A(u) \sin v+B(u) \cos v, \\
-A(u) \cos v+B(u) \sin v, \\
C(u)
\end{array}\right)
$$

where

$$
\begin{aligned}
& A(u)=-\frac{1}{\left(h^{2}-u^{3} g^{\prime} g^{\prime \prime}\right)^{3}}\left(\begin{array}{c}
3 h^{3} u^{4} g^{\prime}+5 h^{3} u^{5} g^{\prime \prime}-h u^{8} g^{\prime} g^{\prime \prime^{2}} \\
+h u^{9} g^{\prime \prime^{3}}+h^{3} u^{6} g^{\prime \prime \prime}+h u^{8} g^{\prime^{2}} g^{\prime \prime \prime}
\end{array}\right), \\
& B(u)=\frac{1}{\left(h^{2}-u^{3} g^{\prime} g^{\prime \prime}\right)^{3}}\left(\begin{array}{c}
-3 h^{2} u^{5} g^{\prime^{2}}-5 h^{2} u^{6} g^{\prime} g^{\prime \prime}+u^{9} g^{\prime^{2}} g^{\prime \prime^{2}} \\
-u^{10} g^{\prime} g^{\prime \prime^{3}}-h^{2} u^{7} g^{\prime} g^{\prime \prime \prime}-u^{9} g^{\prime^{3}} g^{\prime \prime \prime}
\end{array}\right), \\
& C(u)=\frac{1}{\left(h^{2}-u^{3} g^{\prime} g^{\prime \prime}\right)^{3}}\left(\begin{array}{c}
-2 h^{4} u^{3} g^{\prime}-3 h^{2} u^{5} g^{\prime^{3}}-4 h^{4} u^{4} g^{\prime \prime}-7 h^{2} u^{6} g^{\prime^{2}} g^{\prime \prime}-h^{2} u^{7} g^{\prime} g^{\prime \prime^{2}} \\
+2 u^{9} g^{\prime^{3}} g^{\prime \prime^{2}}-h^{2} u^{8} g^{\prime \prime^{3}}-h^{4} u^{5} g^{\prime \prime \prime}-2 h^{2} u^{7} g^{\prime^{2}} g^{\prime \prime \prime}-u^{9} g^{\prime^{4}} g^{\prime \prime \prime}
\end{array}\right) \text {. }
\end{aligned}
$$

We observe that $A(u)=-h g^{\prime}(u) B(u)$. Equation (6.1) by means of (6.2) rise to the following system of ordinary differential equations

$$
\begin{aligned}
\Delta^{\mathrm{III}}(u \cos v) & =\lambda_{1}(u \cos v), \\
\Delta^{\mathrm{III}}(u \sin v) & =\lambda_{2}(u \sin v), \\
\Delta^{\mathrm{III}}(g(u)+h v) & =\lambda_{3}(g(u)+h v),
\end{aligned}
$$

where $\lambda_{1}, \lambda_{2}$ and $\lambda_{3} \in \mathbb{R}$. This means that $\mathbf{M}$ is at most of 3-type. Equation (6.6) becomes

$$
\begin{aligned}
B(u)\left(-h g^{\prime} \sin v+\cos v\right) & =\lambda_{1}(u \cos v), \\
B(u)\left(h g^{\prime} \cos v+\sin v\right) & =\lambda_{2}(u \sin v), \\
C(u) & =\lambda_{3}(g(u)+h v) .
\end{aligned}
$$

First of all, we assume that $\mathbf{M}$ satisfies the condition $\Delta^{\mathrm{III}} \mathbf{x}=0$, thus we have $\lambda_{1}=\lambda_{2}=\lambda_{3}=0$. In this case (6.7) can be written as

$$
B(u)\left(-h g^{\prime} \sin v+\cos v\right)=0
$$




$$
\begin{aligned}
B(u)\left(h g^{\prime} \cos v+\sin v\right) & =0, \\
C(u) & =0 .
\end{aligned}
$$

Since the functions $\cos v, \sin v$ and the constant function are linearly independent, by (6.5) and (6.8), we get

$$
\begin{aligned}
& B(u)=0, \\
& C(u)=0
\end{aligned}
$$

or

$$
\begin{gathered}
-3 h^{2} u^{5} g^{\prime^{2}}-5 h^{2} u^{6} g^{\prime} g^{\prime \prime}+u^{9} g^{\prime^{2}} g^{\prime \prime^{2}}-u^{10} g^{\prime} g^{\prime \prime^{3}}-h^{2} u^{7} g^{\prime} g^{\prime \prime \prime}-u^{9} g^{\prime^{3}} g^{\prime \prime \prime}=0, \\
-2 h^{4} u^{3} g^{\prime}-3 h^{2} u^{5} g^{\prime^{3}}-4 h^{4} u^{4} g^{\prime \prime}-7 h^{2} u^{6} g^{\prime^{2}} g^{\prime \prime}-h^{2} u^{7} g^{\prime} g^{\prime \prime^{2}} \\
+2 u^{9} g^{\prime^{3}} g^{\prime \prime^{2}}-h^{2} u^{8} g^{\prime \prime^{3}}-h^{4} u^{5} g^{\prime \prime \prime}-2 h^{2} u^{7} g^{\prime^{2}} g^{\prime \prime \prime}-u^{9} g^{\prime^{4}} g^{\prime \prime \prime}
\end{gathered}
$$

Differential equations (6.9) and (6.10) admit the particular solution $g(u)=c_{1}$, where $c_{1} \in$ $\mathbb{R}$. In this case, $\mathbf{M}$ is parametrized by

$$
\mathbf{x}(u, v)=\left(u \cos v, u \sin v, c_{1}+h v\right) .
$$

From (6.7), we easly get

$$
\begin{aligned}
& B(u)=\frac{\lambda_{1}(u \cos v)}{\left(-h g^{\prime} \sin v+\cos v\right)}, \\
& B(u)=\frac{\lambda_{2}(u \sin v)}{\left(h g^{\prime} \cos v+\sin v\right)} .
\end{aligned}
$$

and

$$
C(u)=\lambda_{3}(g(u)+h v) .
$$

We discuss two cases according to the values of $\lambda_{i}$.

Case 1: Let $\lambda_{1}=0$ or $\lambda_{2}=0, \lambda_{3} \neq 0$, from (6.11) and (6.12), there are no any suitable solutions.

Case 2: If $\lambda_{1} \neq 0$ or $\lambda_{2} \neq 0, \lambda_{3}=0$, from (6.13), there are no any suitable solutions.

Definition 2. A surface of in the three dimensional simple isotropic space is said to be IIIharmonic if it satisfies the condition $\Delta^{\mathrm{III}} \mathbf{x}=\mathbf{0}$.

Corollary 5. Helicoidal surface given by (3.2) in the three dimensional simply isotropic space $\square_{3}^{1}$ is III-harmonic surface if $g(u)=c_{1}$ for some $c_{1} \in \mathbb{R}$. 
Theorem 2 (Classification). Let $\mathbf{M}$ be a non III-harmonic helicoidal surfaces with non-degenerate second fundamental form given by (3.2) in the three dimensional simply isotropic space $\square_{3}^{1}$. There are no the surfaces $\mathbf{M}$ satisfies the condition $\Delta^{\mathrm{III}} \mathbf{x}_{i}=\lambda_{i} \mathbf{x}_{i}$, where $\lambda_{i} \in \mathbb{R}, i=1,2,3$.

\section{Acknowledgement}

The authors would like to thank the referees for their valuable comments which helped to improve the manuscript. The second author was supported by Basic Science Research Program through the National Research Foundation of Korea(NRF)funded by the Ministry of Education (2015R1D1A1A01060046).

\section{References}

[1] L. J. Alias, A. Ferrandez and P. Lucas, Surfaces in the 3-dimensional Lorentz-Minkowski space satisfying $\Delta \mathbf{x}=$ Ax + B, Pacific J. Math., 156 (1992), 201-208.

[2] M. E. Aydin, Classification results on surfaces in the isotropic 3-space, http://arxiv.org/pdf/1601.03190.pdf.

[3] M. E. Aydin, A generalization of translation surfaces with constant curvature in the isotropic space, J. Geom, DOI 10.1007/s00022-015-0292-0.

[4] C. Baikoussis and L. Verstraelen, On the Gauss map of helicoidal surfaces, Rend. Sem. Math. Messina Ser. II, 2(16) (1993), 31-42.

[5] M. Bekkar, Surfaces of Revolution in the 3-Dimensional Lorentz-Minkowski Space Satisfying $\Delta \mathbf{x}^{i}=\lambda^{i} \mathbf{x}^{i}$,Int. J. Contemp. Math. Sciences, 3(2008), 1173-1185.

[6] S. M. Choi, On the Gauss map of surfaces of revolution in a 3-dimensional Minkowski space, Tsukuba J. Math., 19 (1995), 351-367.

[7] S. M. Choi, Y. H. Kim and D. W. Yoon, Some classification of surfaces of revolution in Minkowski 3-space, J. Geom., 104 (2013), 85-106.

[8] B. Y. Chen, A report on submanifold of finite type, Soochow J. Math. 22 (1996), 117-337.

[9] F. Dillen, J. Pas and L. Vertraelen, On surfaces of finite type in Euclidean 3-space, Kodai Math. J., 13 (1990), $10-21$.

[10] F. Dillen, J. Pas and L. Vertraelen, On the Gauss map of surfaces of revolution, Bull. Inst. Math. Acad. Sinica, 18 (1990), 239-246.

[11] O. J. Garay, An extension of Takahashi's theorem, Geom. Dedicata, 34 (1990), 105-112.

[12] Ch. B. Hamed and M. Bekkar, Helicoidal Surfaces in the three-Dimensional Lorentz-Minkowski space satisfying $\Delta \mathbf{r}_{i}=\lambda_{i} r_{i}$, Int. J. Contemp. Math. Sciences, 4(2009), 311-327.

[13] G. Kaimakamis, B. Papantoniou and K. Petoumenos, Surfaces of revolution in the 3-dimensional LorentzMinkowski space satisfying $\Delta^{\mathrm{III}} \mathbf{r}=$ Ar, Bull. Greek Math. Soc., 50 (2005), 75-90.

[14] B. Senoussi and M. Bekkar, Helicoidal surfaces with $\Delta^{J} r=A r$ in 3-dimensional Euclidean space, Stud. Univ. Babes-Bolyai Math., 60(2015), 437-448.

[15] Z. M. Sipus, Translation Surfaces of constant curvatures in a simply isotropic space, Period Math. Hung., 68 (2014), 160-175.

[16] K. Strubecker, Differentialgeometrie des isotropen Raumes III, Flachentheorie, Math. Zeitsch., 48 (1942), 369427.

[17] T. Takahashi, Minimal immersions of Riemannian manifolds, J. Math. Soc. Japan, 18 (1966), 380-385.

[18] D. W. Yoon, Surfaces of Revolution in the three dimensional pseudo-galilean space, Glasnik Matematicki, 48 (2013), 415-428. 
Usak University, Faculty of Sciences and Arts, Department of Mathematics, 1 Eylul Campus, 64200, Usak-TURKEY. E-mail: murat.karacan@usak.edu.tr

Department of Mathematics Education and RINS, Gyeongsang National University Jinju 660-701, South Korea.

E-mail: dwyoon@gnu.ac.kr

Department of Mathematics, Faculty of Arts and Sciences, Erzincan University, 24000 Erzincan, Turkey.

E-mail: skiziltug@erzincan.edu.tr 